\title{
Разработка прецедентного модуля для идентификаиии сигналов при акустико-эмиссионном мониторинге сложных технических объектов
}

\author{
П.Р. Варшавский 1, к.т.н., доиент, VarshavskyPR@mpei.ru \\ P.B. Алехин 1, ассистент, r.alekhin@gmail.com \\ A.В. Кожевников 1, ассистент, antoko@yandex.ru
}

\author{
${ }^{1}$ Наииональный исследовательский университет "Московский энергетический \\ институт", г. Москва, 111250 , Россия
}

В статье рассматриваются актуальные вопросы разработки модуля для идентификации сигналов, получаемых во время акустико-эмиссионного мониторинга сложных технических объектов, использующего рассуждения на основе прецедентов (CBR - Case-Based Reasoning). Прецедентные методы и системы (CBR-системы) активно применяются для решения целого ряда задач в области искусственного интеллекта, например, для моделирования правдоподобных рассуждений, в том числе рассуждений здравого смысла, машинного обучения, интеллектуальной поддержки принятия решений, интеллектуального поиска информации, интеллектуального анализа данных и др.

Хранение и анализ данных акустико-эмиссионного мониторинга сложных технических объектов в цифровом виде позволили обеспечить требуемую скорость и многовариантность обработки данных, которую бумажная технология не могла обеспечить. С ростом количества разнородных данных увеличился объем работы оператора при выполнении качественного анализа поступающей информации. Для повышения эффективности работы оператора предлагается решение задачи распределения и идентификации рассматриваемых данных акустико-эмиссионного мониторинга с помощью программно реализованного прецедентного модуля (CBR-системы).

C использованием разработанного в среде MS Visual Studio на языке C\# CBR-модуля для идентификации сигналов акустической эмиссии проведены вычислительные эксперименты по оценке эффективности предлагаемых в работе решений на реальных экспертных данных, полученных в результате выполнения акустико-эмиссионного мониторинга металлических конструкций.

Ключевые слова: прецедентный подход, мониторинг, анализ данных, акустическая эмиссия.

Для оценки состояния сложных промышленных объектов активно используют мониторинг - постоянное наблюдение за техническим состоянием конструкции или агрегата. Развитие и распространение систем мониторинга связано со старением оборудования и необходимостью продления срока его эксплуатации, а их успешное функционирование обеспечивается высоким техническим уровнем средств неразрушающего контроля, развитием их алгоритмического и программного обеспечения [1]. Возможность отслеживать возникновение и развитие трещин, разломов и иных дефектов позволяет планировать ремонтные работы или профилактическое обслуживание, а также предотвращать аварийные ситуации.

Наиболее эффективным для мониторинга особо опасных объектов является метод акустической эмиссии (АЭ), под которой понимается явление возникновения и распространения упругих колебаний (акустических волн) в различных процессах, например, при деформа- ции напряженного материала, истечении газов, жидкостей, горении, взрыве и др. [1]. Количественно АЭ - критерий целостности материала, который определяется звуковым излучением материала при его контрольной нагрузке. Эффект АЭ может использоваться для определения образования дефектов на начальной стадии разрушения конструкции и слежения за характером образования и развития дефектов в материале всего объекта в целом.

Основа АЭ-мониторинга - обнаружение и превращение упругих волн в электрический сигнал. Анализ этих сигналов дает ценную информацию о наличии и происхождении дефектов в материале [2]. Одной из проблем, возникающих при проведении АЭ-мониторинга в задаче диагностики состояния контролируемого объекта, является большой объем разнородной диагностической информации: сигналы АЭ, их параметры, показания датчиков температуры, давления, влажности и др. [3]. Помимо этого, при проведении мониторинга контроли- 
руемый объект работает в штатном режиме, но вибрация оборудования, течение продукта (например, жидкость, нефть и др.) в трубопроводе, механические воздействия (например, ослабленные болты или незакрепленные разъемы, которые перемещаются при воздействии ветра, дождь, летящие объекты, пыль) и другие воздействия создают акустические помехи, которые несут в себе заведомо ложную информацию, что затрудняет проведение мониторинга. Для исключения сигналов-помех необходимо решать проблемы идентификации сигналов и фильтрации данных.

Некоторые реальные подходы компенсации влияния фоновой помехи включают в себя изготовление специальных датчиков с электронными фильтрами для блокирования шумов [4], принимая во внимание расстановку датчиков как можно дальше от источника шума и электронную фильтрацию (с помощью либо учета времени появления сигнала, либо различия в спектральном составе истинных сигналов АЭ и фоновых шумов) [3]. Однако даже применение указанных подходов не всегда и не полностью исключает появление шумовых сигналов в данных, предоставляемых эксперту для дальнейшей диагностики. Помимо этого, эксперту зачастую необходимо выбирать из большого числа осциллограмм определенные виды сигналов для анализа дефекта на рассматриваемом объекте.

Для помощи эксперту в решении задачи идентификации сигналов могут применяться методы искусственного интеллекта, в частности, прецедентный подход. Каждый сигнал можно рассматривать как отдельный прецедент, и тогда, имея информацию о ранее выявленных сигналах, можно без активного привлечения эксперта идентифицировать определенные сигналы для их последующего анализа экспертом [5].

\section{Представление информации о сигналах АЭ в виде прецедентов}

В прецедент предлагается включить описание ситуации, решение для данной ситуации и информацию о результате применения решения: $C A S E=$ (Situation, Solution, Result), где Situation - ситуация, к которой применяется данный прецедент; Solution - решение (например, диагноз и рекомендации пользователю); Result - результат применения решения, который может включать список выполненных действий, дополнительные комментарии и ссылки на другие прецеденты, а при необходимости обоснование выбора данного решения и возможные альтернативы [6].

Полученный сигнал содержит достаточно много данных, являющихся промежуточными и не несущих в себе важную информацию. Поэтому отображение сигнала можно упрощенно представить в виде ломаной из экстремумов осциллограммы. Экстремумом будет считаться точка с максимальной/минимальной амплитудой в $\varepsilon$-окрестности, которая определяется длиной сигнала.

По упрощенной версии сигнала строится последовательность возрастаний и спадов сигнала с фиксированием значений относительной амплитуды $\delta A_{i}=\frac{A_{i}}{A_{\max }}$ и диапазона времени. Другими словами, строится последовательность $r_{1}, r_{2}, \ldots, r_{K}$, каждый член которой $r_{i}$ представлен четверкой $\left\{T, \delta A, t_{\mathrm{H}}, t_{\mathrm{\kappa}}\right\}$, где $T$ - тип (рост или спад), $\delta A \in[-1 ; 1]-$ относительная амплитуда на этом участке, $t_{\mathrm{H}}, t_{\mathrm{\kappa}} \in[0 ; 1]$ - относительные временные границы диапазона времени.

Далее полученная последовательность проходит процедуру группировки по следующим правилам:

- пара возрастания и спада сигнала, действующая в пределах $\leq \varepsilon$, считается пиком;

- пара возрастания и спада сигнала, действующая в пределах $>\varepsilon$, считается колебанием;

- идущие подряд $N>1$ колебаний с близкими значениями относительной амплитуды группируются (объединяются в одно с суммарным диапазоном времени).

После группировки образуется аналогичная последовательность $S_{1}, S_{2}, \ldots, S_{L}$, где $S_{i}$ представляется такой же четверкой $\left\{T, \delta A, t_{\mathrm{H}}, t_{\mathrm{\kappa}}\right\}$, но только $T \in\{$ пик; колебания $\}$.

Формирование данной последовательности позволяет значительно сократить информацию о сигнале, что, в свою очередь, ведет к существенному уменьшению временных затрат на поиск похожих сигналов в базе знаний (Б3).

В качестве иллюстрации приведем следующий пример: для сигнала (рис. 1а) длиной в 2000 точек была получена последовательность из 30 четверок (рис. 1б). В графическом отображении сформированной последовательности (рис. 1в) серым цветом показаны колебания, черным - пики.

Полученную последовательность можно сохранить в БЗ как прецедент для дальнейшего 


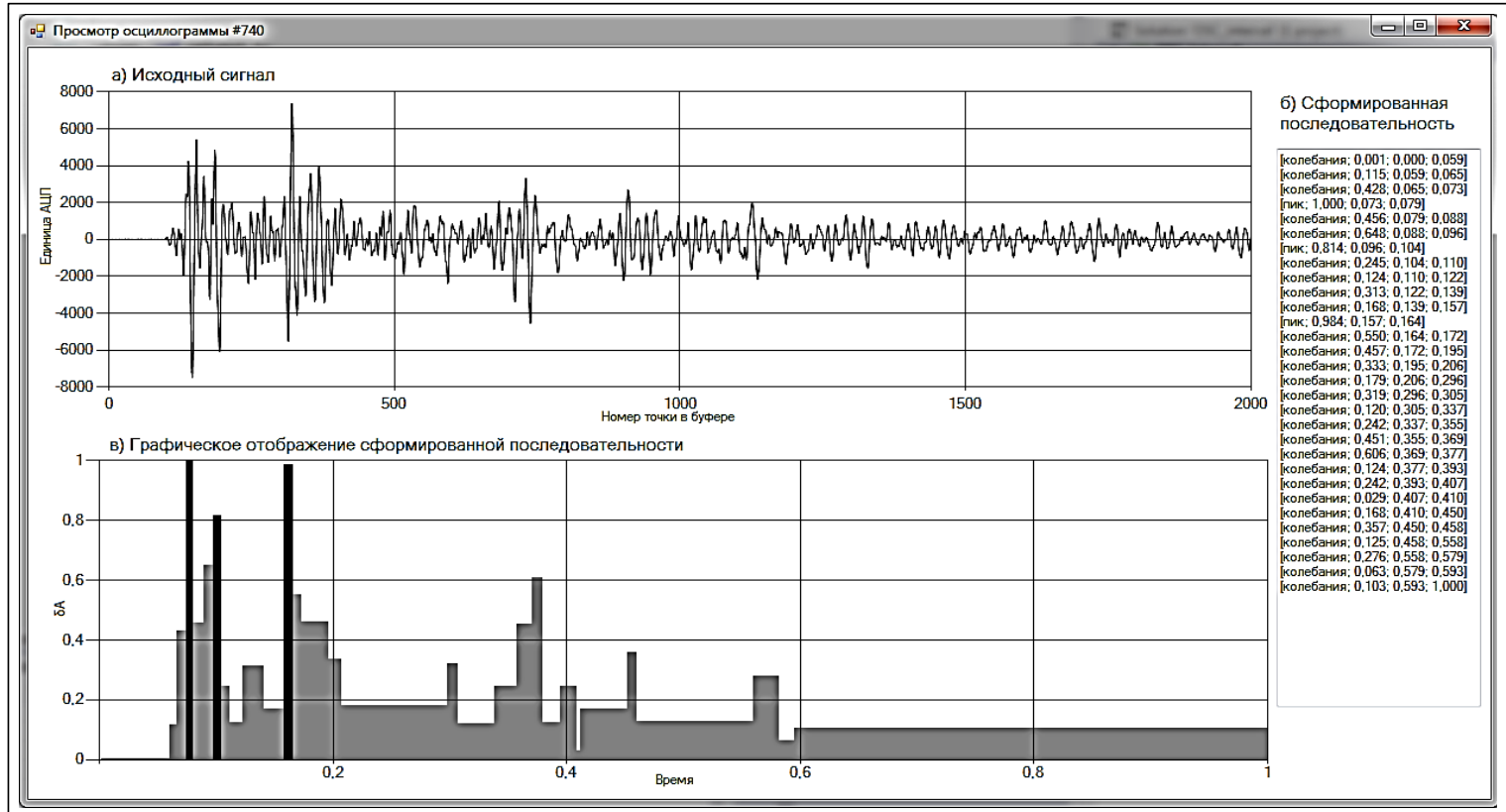

Рис. 1. Пример преобразования данных осииллограммы в прецедент

Fig. 1. An example of converting oscillogram data into a use case

сравнения с имеющимися прецедентами в Б3, в которой могут храниться прецеденты как с сигналами-помехами, так и с актуальными сигналами.

Заметим, что прецедент необязательно содержит полную информацию о сигнале (например, могут отсутствовать конкретные данные осциллограммы сигнала), но он должен содержать информацию, необходимую для экспертной оценки актуальности сигнала [7].

\section{Прецедентный подход для идентификации сигналов АЭ}

CBR-методы поиска решения включают четыре основных этапа, образующих так называемый CBR-цикл: извлечение, повторное использование, адаптация и сохранение прецедента [8]. Существует целый ряд методов извлечения прецедентов, например, метод ближайшего соседа, на основе деревьев решений, метод извлечения с учетом применимости прецедента и др. [6].

Для определения сходства текущего сигнала с прецедентами из Б3 предлагается использовать метод ближайшего соседа (nearest neighbor) [9] - наиболее используемый метод сравнения и извлечения прецедентов. Он позволяет достаточно просто вычислить степень сходства текущей проблемной ситуации и прецедентов из Б3. Для определения степени сходства на множестве параметров, используемых для описания прецедентов и текущей ситуации, вводится некоторая метрика. Далее в соответствии с выбранной метрикой определяется расстояние от целевой точки, соответствующей текущей проблемной ситуации, до точек, представляющих прецеденты из БЗ, и выбирается точка, ближайшая к целевой.

Эффективность метода ближайшего соседа во многом зависит от выбора метрики (меры сходства). Две последовательности могут различаться как самими членами, так и их количеством, поэтому для сравнения последовательностей колебаний выбрана метрика на основе расстояния Левенштейна [9].

Расстояние Левенштейна для последовательностей $S_{1}$ и $S_{2}$ (длиной $M$ и $N$ соответственно) можно подсчитать по формуле $d\left(S_{1}, S_{2}\right)=D(M, N)$, где

$D(i, j)=\left\{\begin{array}{l}0, \quad i=0, j=0, \\ i, \quad j=0, i>0, \\ j, \quad i=0, j>0 \\ \min \left(\begin{array}{l}D(i, j-1)+1 \\ D(i-1, j)+1 \\ D(i-1, j-1)+m\left(S_{1}[i], S_{2}[j]\right)\end{array}\right), j>0, i>0,\end{array}\right.$

где $S_{1}, S_{2}$ - входные последовательности; $m(a, b)=0$, если $a=b$, в противном случае $m(a, b)=1$.

Между новым прецедентом $T$ и сохраненными в базе прецедентами $C$ в выбранной метрике определяются расстояния $d_{C T}$. Для опреде- 
ления значения степени сходства $\operatorname{Sim}(C, T)$ необходимо найти максимальное расстояние $d_{\max }$ в выбранной метрике, используя границы диапазонов соответствующих параметров [7].

Оценка по методу ближайшего соседа: $\operatorname{Sim}(C, T)=1-\frac{d_{C T}}{d_{\text {max }}}$, где $d_{C T}-$ расстояние между текущим сигналом и прецедентом из БП; $d_{\max }-$ максимальное расстояние в выбранной метрике.

При определении класса сигнала вместо конкретных чисел эксперт сравнивает образы сигналов, в частности, наличие колебаний и их амплитуду. Предложенный подход к построению последовательности колебаний для нового прецедента и поиск похожего прецедента в БЗ позволяют ускорить и упростить работу эксперта при идентификации сигналов во время анализа файла данных осциллограмм.

\section{Архитектура прецедентного модуля для идентификации сигналов АЭ}

Разработанный CBR-модуль (рис. 2) является надстройкой, которую в виде отдельной библиотеки можно подключать к программным системам соответствующего назначения, реализованным на различных языках программирования. Модуль может получать от основной программы буфер с данными осцилло- граммы, который далее поступает в блок извлечения осциллограмм и проходит стадии CBRцикла.

Из данных осциллограммы формируется прецедент, далее полученный прецедент проходит стадии извлечения и сохранения в БЗ. Сохраненные прецеденты проходят процедуру маркировки в зависимости от заданных пользователем классов. Однако с ростом числа сохраненных прецедентов в БЗ появляется проблема снижения быстродействия системы [10], для решения которой применяется блок сокращения числа прецедентов.

CBR-модуль решает задачу маркировки и фильтрации файла данных осциллограмм для облегчения и ускорения работы эксперта (оператора). После анализа содержимого файла данных с помощью интерфейса модуль передает основной программе номера интересующих (маркированных положительно) осциллограмм, что позволяет оператору быстро перемещаться по файлу с данными АЭ-мониторинга.

\section{Взаимодействие между разработанным модулем и основной программой}

Разработан набор сообщений, с помощью которых идет общение между основной программой АЭ-мониторинга и реализованной

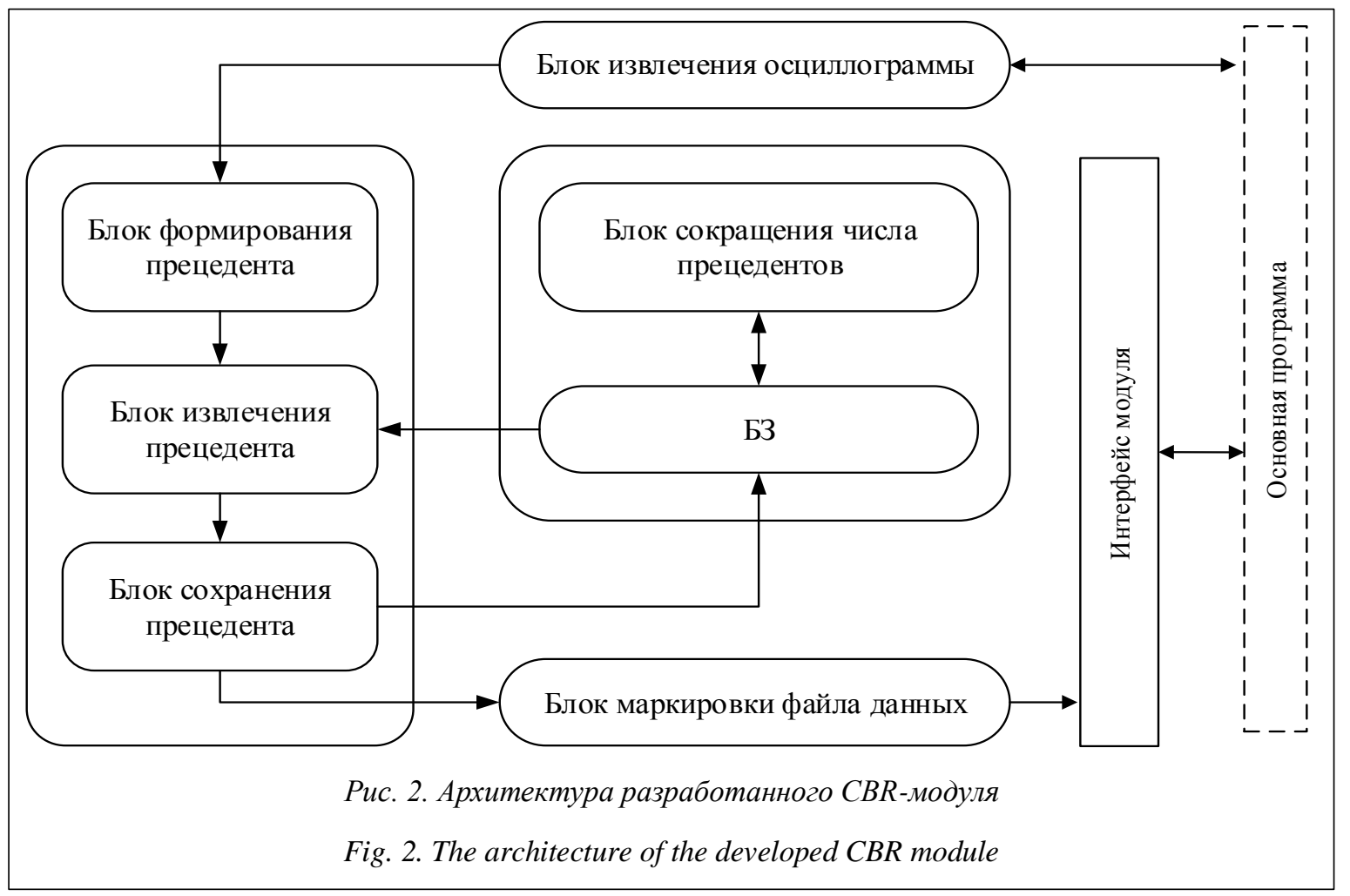


надстройкой. Для начала работы CBR-модуля необходимо загрузить конфигурацию с подобранными и помеченными экспертом осциллограммами. После этого модуль готов к приему сообщений с осциллограммами для идентификации. Сообщения должны содержать в себе следующую информацию: размер буфера (в байтах), размер единицы данных (в байтах), номер осциллограммы, начало блока точек осциллограммы (опционально), количество точек осциллограммы (опционально).

Если последние два пункта содержат в себе нулевое значение, то из буфера извлекается вся осциллограмма, в противном случае извлекается указанный набор точек. Извлеченный набор сохраняется как массив вещественных чисел (double) и проходит обработку прецедента, реализуя CBR-цикл. Соответствующий алгоритм представлен на рисунке 3. После обработки в БЗ CBR-модуля сохраняются сформированная последовательность, результат маркировки и полученный номер осциллограммы.

Модуль может принимать сообщения, в которых будут указаны классы осциллограмм, требующие просмотра и изучения оператором. В этом случае происходит настройка приоритетных для отображения классов маркированных осциллограмм.

Номера осциллограммы используются для быстрого перемещения по имеющимся в основной программе данным. Необходимо отправить сообщение, в котором будут содержаться текущий номер осциллограммы основного проекта и направление поиска ближайшей подходящей по маркировке осциллограммы. Таким образом, пользователь не ограничен в перемещении по файлу данных.

В случае, когда пользователя не устраивает результат, полученный CBR-модулем, можно отправить сообщение об изменении класса маркировки.

\section{Программная реализация прецедентного модуля для идентификации сигналов АЭ}

Предложенный прецедентный подход для идентификации сигналов АЭ на основе метода ближайшего соседа с использованием метрики Левенштейна был реализован в программном модуле для системы анализа файлов, содержащих данные АЭ-мониторинга (рис. 4), на языке C\# (.NET 4.5) [11] в среде Microsoft Visual Studio 2012 под операционную систему MS Windows.

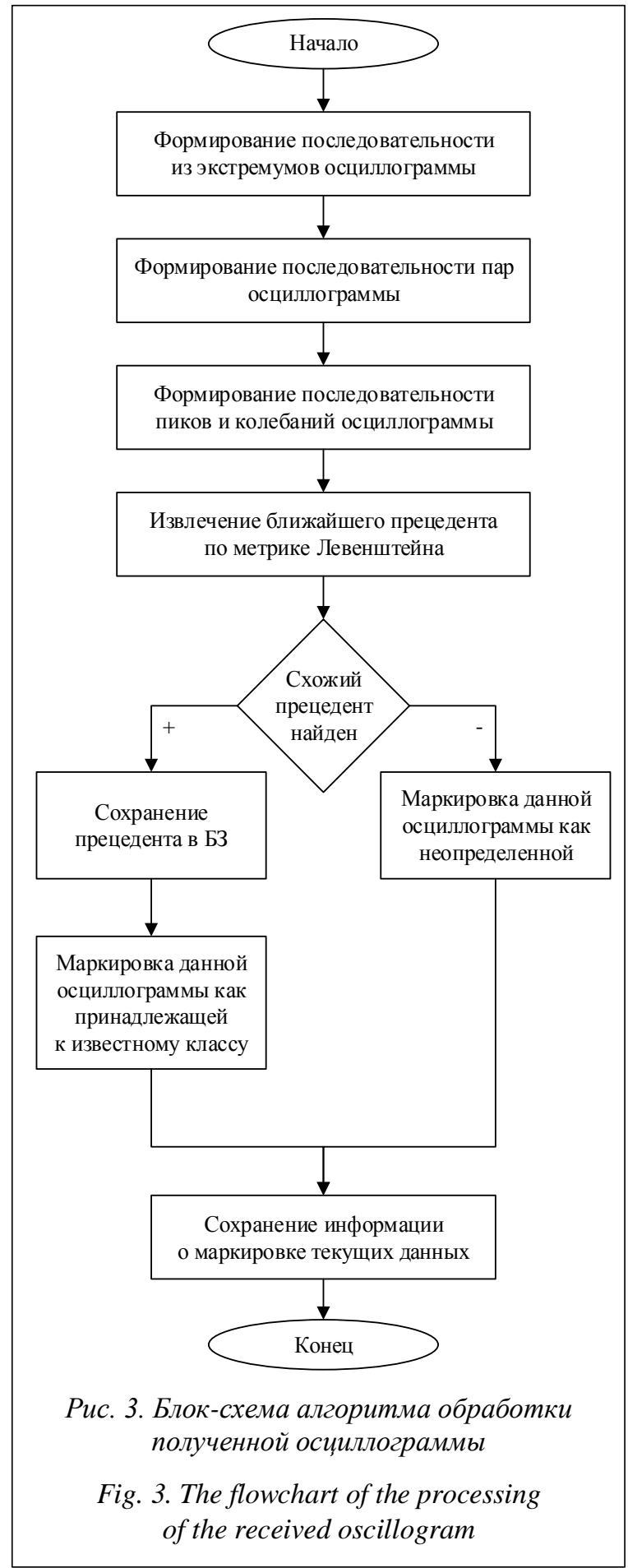

Пользователь задает настройки работы CBR-модуля: устанавливает виды уже известных (классифицированных экспертом) сигналов и выбирает необходимые для отображения классы сигналов. После этого CBR-модуль выполняет процедуру маркировки файла данных и предоставляет пользователю упрощенное перемещение по содержимому файла, позволяющее просматривать только заданные пользова- 


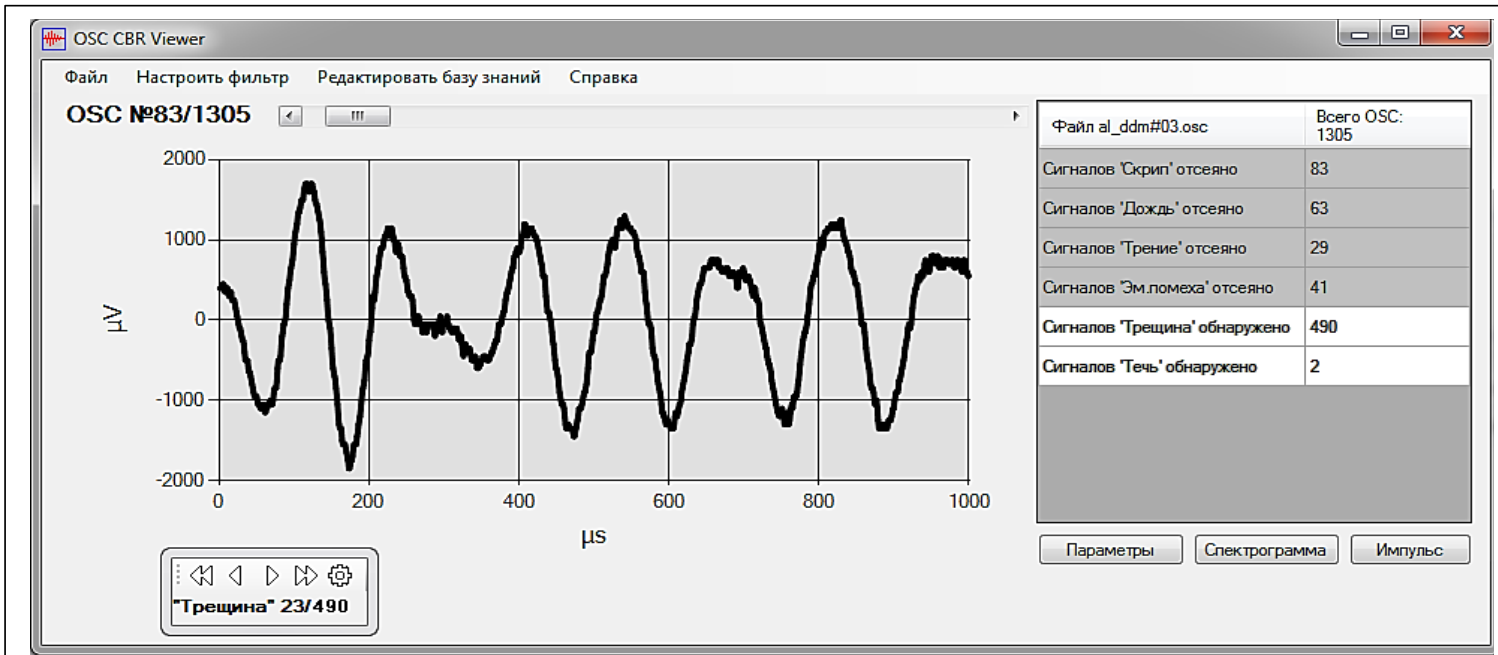

Pис. 4. Пример работы CBR-модуля

Fig. 4. A CBR module operation example

телем классы сигналов. При желании можно совмещать обычный и упрощенный просмотры для детального анализа файла данных.

Работа модуля была проверена экспертами на тестовых данных, полученных при АЭ-мониторинге металлических конструкций (более 20 файлов измерений), результаты подтвердили эффективность применения прецедентного подхода для идентификации сигналов, получаемых во время АЭ-мониторинга сложных технических объектов. Применение реализованного CBR-модуля позволяет значительно сократить время анализа данных благодаря полученной на основе прецедентов маркировке содержимого файла (более $10^{6}$ осциллограмм).

\section{Заключение}

В работе исследована возможность применения методов поиска решения на основе прецедентов для идентификации сигналов АЭмониторинга сложных технических объектов. Для извлечения прецедентов из Б3 (базы прецедентов) предложено использовать метод ближайшего соседа и расстояние Левенштейна в качестве меры сходства. Разработан метод представления сигналов АЭ-мониторинга как прецедента. На основе предложенного подхода реализован прецедентный модуль для идентификации сигналов АЭ-мониторинга. Работа модуля была проверена на тестовых данных, полученных при АЭ-мониторинге металлических конструкций. Полученные результаты показали эффективность предложенного подхода и его реализации в виде CBR-модуля.
Работа выполнена при финансовой поддержке проектов РФФИ №№ 18-01-00459, 18-29-03088, 18-51-00007.

\section{Литература}

1. Барат В.А., Алякритский А.Л. Метод статистической обработки данных акустико-эмиссионного мониторинга на примере реактора гидроочистки Мозырского НП // В мире НК. 2008. № 4. C. 52-55.

2. Терентьев Д.А. Идентификация сигналов акустической эмиссии при помощи частотно-временного анализа // В мире НК. 2013. № 2. С. 51-55.

3. Kwon O.-Y., Ono K. Acoustic emission characterization of the deformation and fracture of an sicreinforced aluminum matrix composite. JAE, 1990, vol. 9, pp. 123-129.

4. Кузьмин А.Н., Журавлев Д.Б., Филиппов С.Ю. Коррозия - приговор или диагноз? К вопросу технической диагностики тепловых сетей // Технадзор. 2009. № 3. С. 32-35.

5. Alekhin R.V., Varshavsky P.R., Eremeev A.P., Kozhevnikov A.V. Application of the casebased reasoning approach for identification of acoustic-emission control signals of complex technical objects. Proc. 3rd Russian-Pacific Conf. on Computer Technology and Applications (RPC), 2018, pp. 28-31. DOI: 10.1109/RPC.2018.8482124.

6. Еремеев А.П., Варшавский П.Р., Куриленко И.Е. Моделирование временных зависимостей в интеллектуальных системах поддержки принятия решений на основе прецедентов // Information Technologies and Knowledge. 2012. T. 6. № 3. С. 227-239 (рус.).

7. Варшавский П.Р., Алехин Р.В., Кожевников А.В. Разработка прецедентного модуля для идентификации сигналов при акустико-эмисси- 
онном контроле сложных технических объектов // КИИ-2018: тр. XVI Национальн. конф. 2018. T. 2. C. 222-229.

8. Aamodt A., Plaza E. Case-based reasoning: foundational issues, methodological variations, and system approaches. Artificial Intelligence Communications. 1994, vol. 7, no. 1, pp. 39-59.

9. Рассел С., Норвиг П. Искусственный интеллект: современный подход; [пер. с англ.]. М.:
Вильямс, 2007. 1408 с.

10. Варшавский П.Р., Ар Кар Мьо, Шункевич Д.В. Применение методов классификации и кластеризации для повышения эффективности работы прецедентных систем // Программные продукты и системы. 2017. № 4. С. 625-631.

11. Albahari J., Albahari B. C\# 4.0 in a Nutshell. The Definitive Reference. O'Reilly Media, Inc., 2010, 1056 p.

\section{Development of the case-based reasoning module for identification of acoustic-emission monitoring signals of complex technical objects}

\section{P.R. Varshavsky ${ }^{1}$, Ph.D. (Engineering), Associate Professor, VarshavskyPR@mpei.ru}

R.V.Alekhin ${ }^{1}$, Assistant, r.alekhin@gmail.com

A.V.Kozhevnikov ${ }^{1}$, Assistant, antoko@yandex.ru

${ }^{1}$ Nationa Research University “MPEI”, Moscow, 111250, Russia

Abstract. The paper examines important issues of developing a module for identifying signals obtained during acoustic emission monitoring of complex technical objects using case-based reasoning (CBR). Casebased methods and systems are used to solve a number of artificial intelligence problems (for example, for modeling plausible reasoning (common sense reasoning), machine learning, intellectual decision support, intelligent information search, data mining and etc.).

The storage and analysis of acoustic emission monitoring data of complex technical objects in digital form made it possible to ensure the required speed and multivariate data processing, which the paper technology could not provide. As the amount of heterogeneous data grows, the amount of work for an operator in qualitative analyzing has increased. To improve the efficiency of an operator, it is proposed to solve the problem of distributing and identifying the acoustic emission monitoring data by CBR tools.

The CBR module for identifying acoustic emission signals has been developed in C\# using MS Visual Studio. In order to evaluate the effectiveness of the proposed solutions, the paper shows the results of computational experiments on real expert data obtained from acoustic emission monitoring of metal constructions.

Keywords: case-based approach, monitoring, data analysis, acoustic emission.

Acknowledgements. This work was supported by RFBR projects no. 18-01-00459, 18-29-03088, 18-51-00007.

\section{References}

1. Barat V.A., Alyakritski A.L. Statistical data processing of acoustic emission monitoring: Mozyrski NPZ hydrotreater case study. NDT World. 2008, no. 4, pp. 52-55 (in Russ.).

2. Terentev D.A. Time frequency analysis for identification of acoustic emission signals. NDT World. 2013, no. 2, pp. 51-55 (in Russ.).

3. Kwon O.-Y., Ono K. Acoustic emission characterization of the deformation and fracture of an sic-reinforced aluminum matrix composite. JAE. 1990, vol. 9, pp. 123-129.

4. Kuzmin A.N., Zhuravlev D.B., Filippov S.Yu. Corrosion - sentence or diagnosis? On the issue of technical diagnostics of heat networks. Engineering Supervision. 2009, no. 3, pp. 32-35 (in Russ.).

5. Alekhin R.V., Varshavsky P.R., Eremeev A.P., Kozhevnikov A.V. Application of the case-based reasoning approach for identification of acoustic-emission control signals of complex technical objects. Proc. 3rd Russ.-Pacific Conf. on Computer Technology and Applications (RPC). 2018, pp. 28-31 (in Russ.). DOI: 10.1109/RPC.2018.8482124.

6. Eremeev A.P., Varshavsky P.R., Kurilenko I.E. Modeling the time-dependent in intellectual systems of decision making support on the basis of precedents. Information Technologies \& Knowledge. 2012, vol. 6, no. 3, pp. 227-239 (in Russ.).

7. Varshavsky P.R., Alekhin R.V., Kozhevnikov A.V. Development of the precedent module for identification of signals with acoustic-emission control of complex technical objects. Proc. 16th Russ. Conf. (RCAI 2018). Moscow, 2018, no. 2, pp. 222-229 (in Russ.).

8. Aamodt A., Plaza E. Case-based reasoning: foundational issues, methodological variations, and system approaches. Artificial Intelligence Communications. IOS Press, 1994, vol. 7, no. 1, pp. 39-59.

9. Russell S., Norvig P. Artificial Intelligence: Modern Approach. Prentice Hall Publ., 2 ed., 2002, 1132 p. (Russ. ed.: 2nd ed., Moscow, Villiams Publ., 2006, 1408 p.).

10. Varshavsky P.R., Ar Kar Myo, Shunkevich D.V. Classification and clustering methods for improving efficiency of case-based systems. Software \& Systems. 2017, no. 4, pp. 625-631 (in Russ.).

11. Albahari J., Albahari B. C\# 4.0 in a Nutshell. The Definitive Reference. O'Reilly Media, 2010, 1056 p. 